\title{
1 A heterohexameric protein consisting of six linked 2 single-domain antibodies is highly protective for BoNT/A, BoNT/B and BoNT/E exposures
}

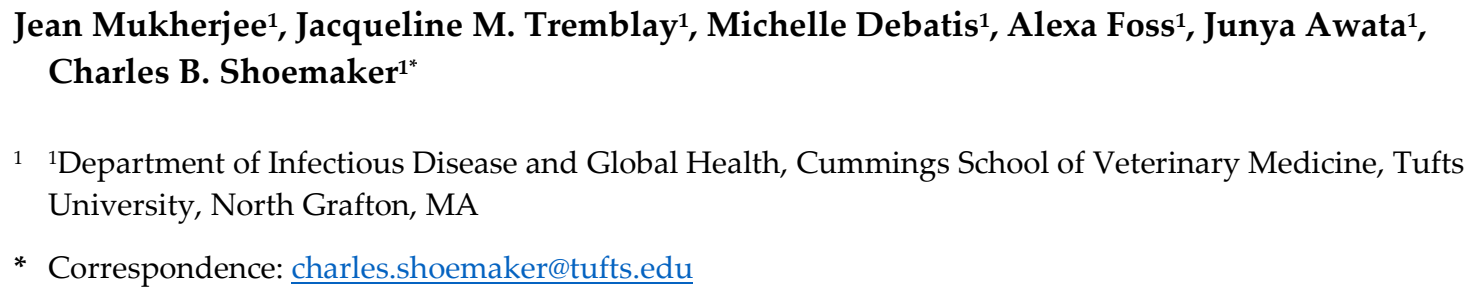

Received: date; Accepted: date; Published: date

\begin{abstract}
Botulinum neurotoxin (BoNT) serotypes A, B and E cause the vast majority of human botulism cases and pose the greatest bioterrorism threats. We previously identified multiple camelid single-domain antibodies (VHHs) that each neutralize BoNT/A, BoNT/B or BoNT/E. We also demonstrated that heterodimers of linked toxin-neutralizing $\mathrm{VHHs}$ are much more potent than VHH monomer pools in preventing BoNT intoxication. In this study, we expressed two different heterohexamer proteins (VNA1-ABE and VNA2-ABE) of $~ 100 \mathrm{kDa}$ secreted from mammalian host cells, each containing the same six linked anti-BoNT VHH components ordered in two different combinations. Each heterohexamer contained two VHHs that neutralize BoNT/A, BoNT/B or BoNT/E. Both heterohexameric antitoxins displayed similar strong binding properties for the three targeted BoNT serotypes by ELISA. One ug of each heterohexameric antitoxin fully protected groups of mice co-administered with 100 LD 50 of BoNT/A, BoNT/B or BoNT/E, or a pool containing $100 \mathrm{LD}_{50}$ of each of the three toxins. The results demonstrate that long chains of at least six different linked VHHs can be expressed such that all component VHHs in the multimer retain their target binding activities. These findings make more feasible the development of a BoNT antitoxin product consisting of a small pool of proteins that, in combination, neutralize all known BoNT serotypes and subtypes.
\end{abstract}

Keywords: 1, VHH; 2, toxin; 3, antitoxin; 4, Botulinum neurotoxin; 5, BoNT; 6, neutralization

Key Contribution: Heteromultimeric proteins consisting of six linked, VHH antibodies, and including VHHs that neutralize BoNT/A, BoNT/B and BoNT/E, retain high potency to protect mice challenged with high doses of all three of these BoNT serotypes.

\section{Introduction}

Protein toxins are important virulence factors in many important diseases and can also cause harm through accidental exposures or deliberate bioterror incidents. Specific antitoxin antibodies (Abs) are the primary treatment modality for toxin exposures. Traditionally, antitoxins are either elicited in patients through vaccination or passively administered as antiserum or purified Abs. Immunization is not practical for most toxin threats and traditional antitoxin treatments are often expensive, difficult to access and may pose safety concerns. Thus, new therapeutic options that mitigate these issues are needed.

One alternative to conventional serum-based antitoxins are single-domain Abs (sdAbs) generated from camelid [1] or shark [2] heavy-chain only Abs (HcAbs). These simple Abs are the $\mathrm{V}_{\mathrm{H}}$ 
region of HcAbs and are often called nanobodies or VHHs. Advantages of VHHs over conventional Abs are that VHHs are small $(\sim 14 \mathrm{kDa})$ proteins, more stable to $\mathrm{pH}$ and temperature extremes, and can be produced inexpensively in microbial hosts [3-5]. In addition, VHHs can be expressed as multimeric proteins which impart improved antitoxin potencies [6-8] and permit the targeting of multiple toxins with a single protein agent [9-13].

VHH antitoxins also have some disadvantages as compared to conventional antibodies. One disadvantage is that VHHs are much more rapidly cleared from the circulation than conventional antibodies. Since antitoxins are generally administered in a single post-exposure treatment, serum persistence is not important if the treatment adequately neutralizes the toxin that had already entered the system. For situations in which longer serum persistence is needed, the antitoxin VHHs can be fused to a second $\mathrm{VHH}$ or peptide having high affinity for a major serum protein such as albumin $[7,14]$. Alternatively, VHHs are easily amenable to genetic delivery and can be expressed for extended times in patients by gene therapy with viral vectors [7] or delivered by encapsulated synthetic mRNA [15]. A second potential disadvantage of VHHs is that they lack the Fc effector functions of conventional antibodies, which results in the inability of VHHs to promote clearance of the toxin from the circulation. Treatment with polyclonal antisera results in the decoration of the toxin with multiple $\mathrm{Ab}$ Fc domains, and it is known that decoration by at least three Fc domains leads to rapid serum clearance [16]. Promotion of toxin serum clearance, though, should not be necessary if the antitoxin agents adequately neutralize the toxin until natural processes clear the toxin. Indeed, HBAT (heptavalent botulinum antitoxin, Cangene Corporation), which is the current treatment for exposure to Clostridium botulinum neurotoxins (BoNT), consists of polyclonal Fab domains purified from horse antiserum. Thus HBAT, like VHHs, lacks Fc domains, resulting in rapid product clearance from serum [17] and an inability to promote Fc receptor-mediated toxin clearance. For situations in which an Fc domain is necessary for a VHH-based antitoxin product to be fully effective, it is possible to produce the VHH agents as fusions to Fc domains [18] or to coadminister an anti-tag effector $\mathrm{Ab}$ which binds to peptide tags engineered into the $\mathrm{VHH}$ products $[8,11,19]$ and thus provide Fc functions.

Most protein toxin threats to humans derive from pathogens that exist in nature as families of related microorganisms that produce natural variant forms of one or more toxins. BoNTs are an extreme example of this challenge as at least seven distinct BoNT serotypes have been identified [20], most of which can be found with diverse natural subtype variations. Antitoxin products must be capable of broadly neutralizing all potential variants of the target toxin. The use of polyclonal antitoxin sera to treat toxin exposures generally provides broad protection from toxin variants since it consists of a diverse pool of antibodies recognizing many different epitopes, at least a few of which are likely to be effective against each of the variants. Efforts to replace polyclonal antitoxin products with clonal antibody products such as VHHs must also be capable of broadly neutralizing natural variants. One solution that is being developed for BoNTs is to create pools of three mAbs for each serotype in which each $\mathrm{mAb}$ has been carefully selected for the ability to broadly neutralize all known natural variant subtypes for the serotype and each binds their target at non-overlapping epitopes. With this design, each $\mathrm{mAb}$ pool should neutralize virtually all natural subtype variants and decorate each toxin with three $\mathrm{mAb} F \mathrm{~F}$ domains so as to also promote rapid toxin clearance from the serum [21-26].

VHH antitoxins may be engineered to become capable of broad neutralization of natural variants through production of heteromultimers containing a series of different linked VHHs, each selected for the ability to broadly neutralize a subset of natural toxin variants. Even when both VHH components of a heterodimer have relatively poor neutralizing activity on a toxin as monomers, the linked VHHs can acquire high potency [11]. Prior studies have demonstrated that VHH heterotetramers with four different toxin neutralizing VHHs can be produced and that each VHH component appears to fold properly during expression and remain functional [9]. These

92 heteromultimeric proteins are called VHH-based neutralizing agents or VNAs. In this study, we 
bioRxiv preprint doi: https://doi.org/10.1101/2021.03.02.433625; this version posted March 2, 2021. The copyright holder for this preprint (which was not certified by peer review) is the author/funder, who has granted bioRxiv a license to display the preprint in perpetuity. It is made available under aCC-BY-NC-ND 4.0 International license.

tested the feasibility of expressing a VHH heterohexameric VNA in which two VHH components each recognize one of three different BoNT serotypes; BoNT/A, BoNT/B and BoNT/E. The heterohexamer components are organized in two different formats and both proteins tested for the ability to bind to each of the three toxins and to protect mice from lethal intoxication by the toxins.

\section{Results}

\subsection{Expression of $V H H$ heterohexamer proteins}

Two synthetic genes, each encoding six linked VHH components, were designed and prepared. Both heterohexameric VNAs contained the same six VHHs arranged in a different order (Figure 1A). The six VHHs selected as components consisted of two VHHs that were each known to potently neutralize one of three different toxins; BoNT/A, BoNT/B or BoNT/E. The two component VHHs that neutralize BoNT/A are ciA-H7 (A1) and ciA-B5 (A2) [19]; for BoNT/B they are JLI-G10 (B1) and JLKG12 (B2) [27]; and for BoNT/E they are, JLE-G6 (E1) and JLE-E9 (E2) [28]. All VHHs in the VNAs recognized non-overlapping neutralizing epitopes on their different toxin targets. The six VHHs were linked in two different combinations as shown in Figure 1A, and the complete monomer and heterohexamer sequences are provided in Figure S1A and S1B. The first heterohexamer construction, VNA1-ABE contains the pairs of VHHs recognizing each BoNT serotype linked together such that the order from amino to carboxyl end would be A1/A2/B1/B2/E1/E2. In VNA1-ABE, all VHHs were linked together by flexible spacers that match the spacers of VHH heterodimers for each BoNT serotype that had been successfully tested as antitoxin in mice [19, 27, 28]. The second VHH heterohexameric VNA construction, VNA2-ABE, contains the pairs of VHHs recognizing each serotype separated from one another by two VHHs targeting a different BoNT serotype, with the order A1/B1/E1/A2/B2/E2. In VNA2-ABE, we employed shorter, 5 amino acid GGGGS spacers to minimize overall protein size.

A
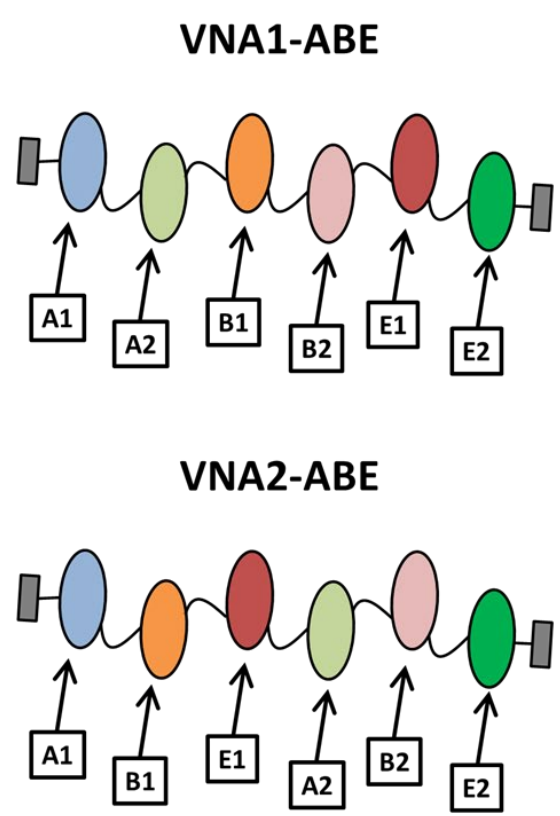

\section{B}

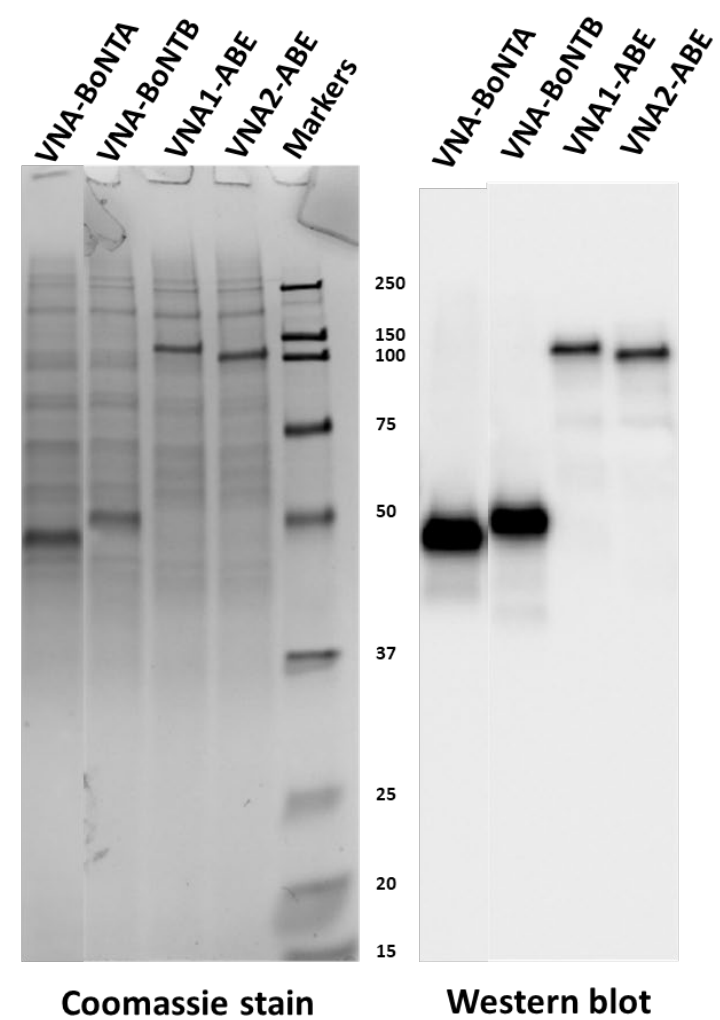

Figure 1. Design and expression of heterohexameric VNA antitoxins. A. Cartoon image showing the VHH component structures of VNA1-ABE and VNA2-ABE. The sequences of the six component VHHs: A1, A2, B1, B2, E1, E2 are shown in Figure S1A. The full amino acid sequences of the two VNA heterohexamers are shown in Figure S1B. B. Conditioned medium was obtained from the $\mathrm{CHO}$ 
cells transfected with vectors encoding the four different indicated VNAs and samples were loaded onto SDS-PAGE for Coomassie staining $(10 \mu \mathrm{l})$ or western blotting $(1 \mu \mathrm{l})$. Western blots were probed with HRP/anti-E-tag, molecular weight marker sizes are shown in kb.

The DNAs encoding VNA1-ABE and VNA2-ABE were ligated into a mammalian cell expression vector, $\mathrm{pSecB}$, under the control of the $\mathrm{CMV}$ promoter and fused to the human Igא leader sequence. Similar pSecB vectors were prepared containing heterodimers of BoNT/A neutralizing VHHs A1/A2 (VNA-BoNTA) and BoNT/B-neutralizing VHHs, B1/B2 (VNA-BoNTB). The plasmids were transfected into $\mathrm{CHO}$ cells grown in serum-free medium and the conditioned medium collected after 3-4 days. The conditioned medium was characterized by SDS-PAGE followed by protein staining or Western blotting (Figure 1B). The products of expected size were visible by protein staining of conditioned medium and their identities confirmed by the blots. The blots also revealed that there were no detectable truncated VNA products of the heterohexamers in the conditioned medium. The expression levels for both heterohexamer proteins were estimated to be approximately $10 \mu \mathrm{g} / \mathrm{ml}(\sim 100$ $\mathrm{nM})$ based on comparisons of the staining intensity against protein standards. Similar protein expression levels were obtained in conditioned media from cells transfected with the two heterodimer VNA vectors $(\sim 10 \mu \mathrm{g} / \mathrm{ml}(\sim 300 \mathrm{nM}))$.

\subsection{VHH heterohexamers recognize each of their three BoNT targets}

Mammalian cell produced recombinant VNA1-ABE and VNA2-ABE VHH hexamer proteins were assessed for their ability to bind to each of the three BoNT serotypes to which the monomer components recognize. ELISAs were performed to assess VNA binding to catalytically inactive forms of BoNT/A, BoNT/B or BoNT/E holotoxins [29, 30]. As shown in Figure 2, each of the two hexameric VNAs recognized all three toxins with approximately equal apparent affinities based on their similar $\mathrm{EC}_{50}$ between $\sim 0.5$ and $2 \mathrm{nM}$. The VNA-BoNTA and VNA-BoNTB heterodimers, also expressed in mammalian cells, possessed similar apparent affinities (EC50s) to the two heterohexamers tested on ciBoNTA and ciBoNTB. The VNA-BoNTE heterodimer, which was expressed in E. coli, had an EC50 similar to the two heterohexamers when tested on ciBoNTE. The EC50 values reflecting apparent affinity likely under-represent the actual affinity of these agents for their native BoNT targets because we employed ELISAs with plastic-coated ciBoNTs so as to be consistent across the three BoNT serotypes. Other studies have shown that VHHs typically recognize the more conformationallynative antibody-captured BoNT targets with higher apparent affinity than the plastic-coated BoNTs [28]. Nevertheless, the results demonstrate that the apparent affinity of the two heterohexamers for their three different BoNT target serotype targets did not detectably differ from that of comparable VHH heterodimers, despite their expression as a single chain of six linked VHH components.

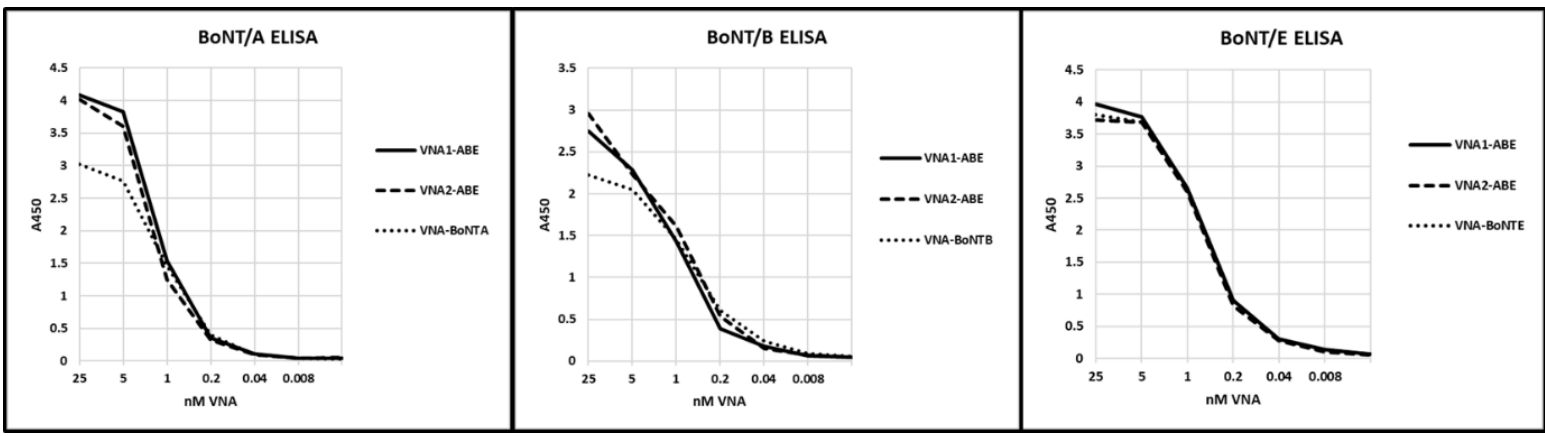

Figure 2. Dilution ELISAs of heterohexamer and heterodimer VNAs on different BoNT serotypes. ELISAs were performed by coating $1 \mu \mathrm{g} / \mathrm{ml}$ of the target BoNT (ciBoNTA, ciBoNTB or ciBoNTE) onto Costar tissue culture 96-well plates. All VNAs were diluted to $25 \mathrm{nM}$ and then subjected to 1:5 serial dilutions. VHH binding was detected with HRP/anti-E-tag reagent. 
Several in vivo mouse studies were performed to assess the antitoxin efficacies of the two heterohexamers. Figure 3 shows survival plots for groups of five mice challenged with 100 LD50 of each BoNT serotype, or a pool containing $100 \mathrm{LD}_{50}$ of each of the three BoNT serotypes. All control mice died $<4$ hours following toxin exposure while all mice given 10 pmoles $(1 \mu \mathrm{g})$ VNA1-ABE or VNA2-ABE survived challenge with either the individual or combined toxin challenge. All treated mice developed transient mild-moderate signs of botulism and recovered within a week. Previous studies have shown that use of a pool of two unlinked BoNT/A-neutralizing VHHs, ciA-H7 (A1) and ciA-B5 (A2) are much less potent as antitoxins than a heterodimer containing the same $\mathrm{VHH}$ components and unable to protect mice challenged with 100 LD50 of BoNT/A [19]. The two BoNT/Eneutralizing monomer component, JLE-G6 (E1) or JLE-E9 (E2), were unable to protect mice from as low as $3 \mathrm{LD}_{50}$ of BoNT/E [28]. These results strongly suggest that the VHH monomer components of the heterohexamer remain linked together within the treated animals.

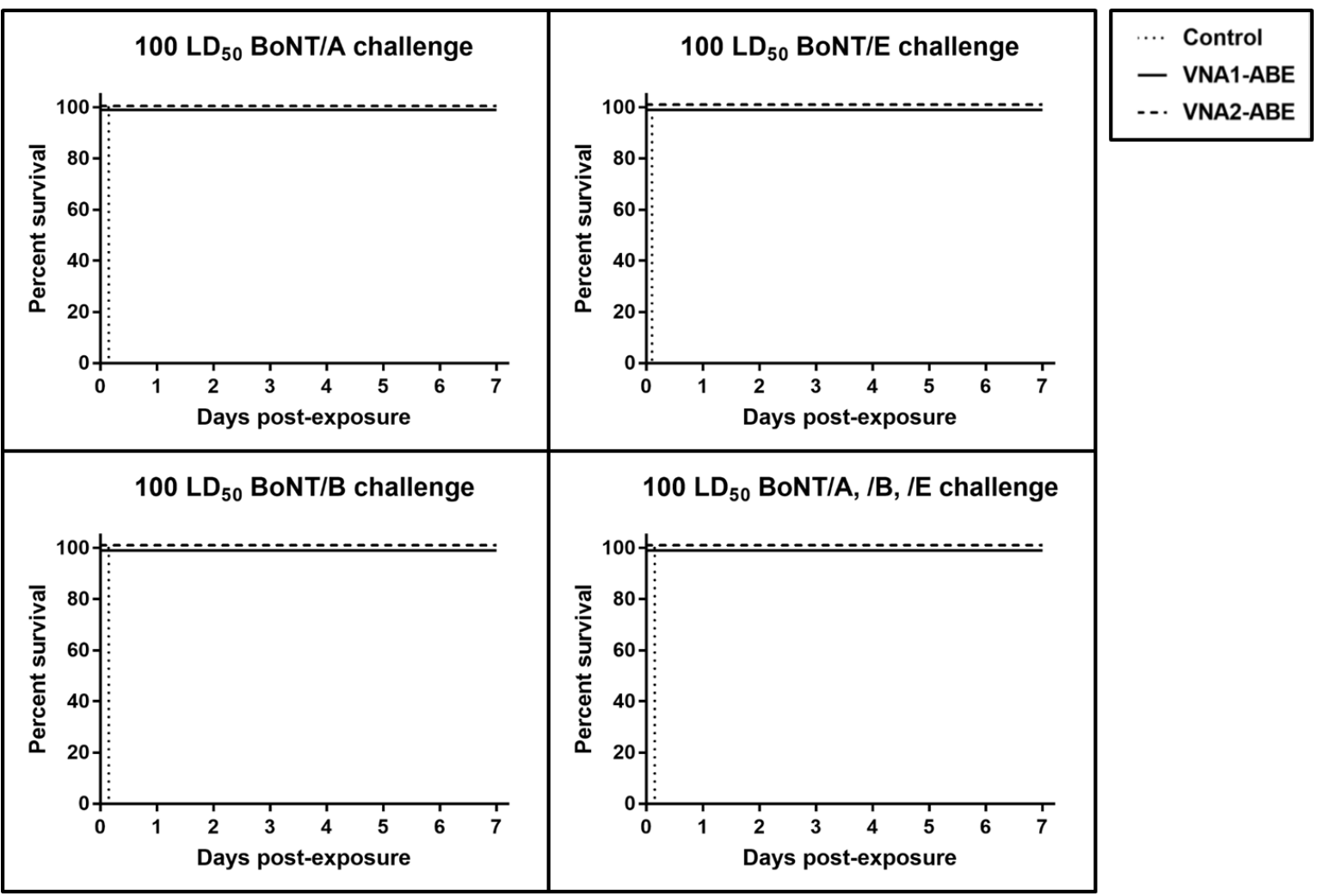

Figure 3. Heterohexamer VNAs protect mice from $100 \mathrm{LD}$ 50 doses of BoNT/A, BoNT/B and BoNT/E. Groups of five mice were co-administered $1 \mu \mathrm{g}$ ( 10 pmoles) of VNA1-ABE or VNA2-ABE and 100 $\mathrm{LD}_{50}$ of BoNT/A, BoNT/B or BoNT/E, or a combination of all three toxins, as indicated. Mice were then monitored for survival and signs of botulism for a week. The survival of mice is plotted as a function of time post-exposure. All VNA-treated groups had 100\% survival, though showing mild signs of botulism, and these outcomes were significantly different from untreated controls $(p<0.001$ by log-rank Mantel-Cox test).

Additional in vivo studies with the heterohexamer proteins compared the survival of mice challenged with $500 \mathrm{LD} 50$ of BoNT/B that were co-administered with heterohexamers VNA1-ABE, VNA2-ABE or the heterodimer, VNA-BoNTB. As shown in Figure 4A, control mice died within two hours while all mice treated with the three VNAs survived, showing only mild signs of botulism before recovering. Finally, groups of mice were challenged with 10,000 LD50 of BoNT/A coadministered with 10 pmoles of heterohexamers VNA1-ABE, VNA2-ABE or 10 pmoles of heterodimer VNA-BoNTA (Figure 4B). Control mice died in less than an hour while mice treated with the VNAs survived about for one or two days. These results suggest there may be small differences in potency between VNA1-ABE and VNA2-ABE revealed at the highest challenge dose, although this would need to be confirmed by additional studies. 
A

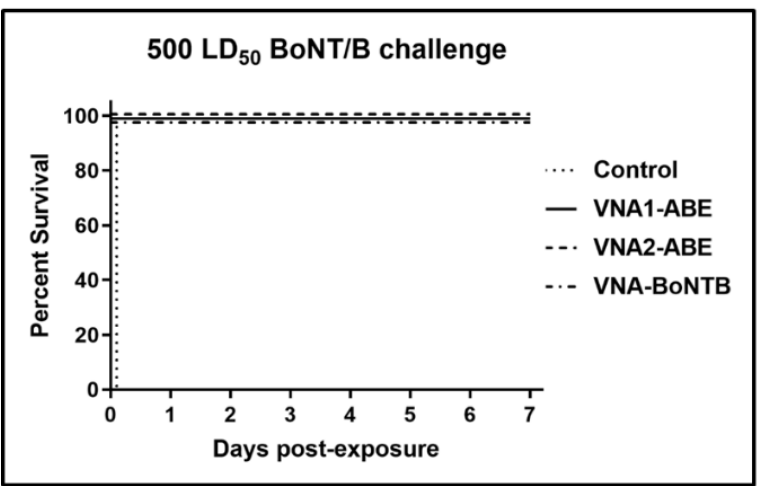

B

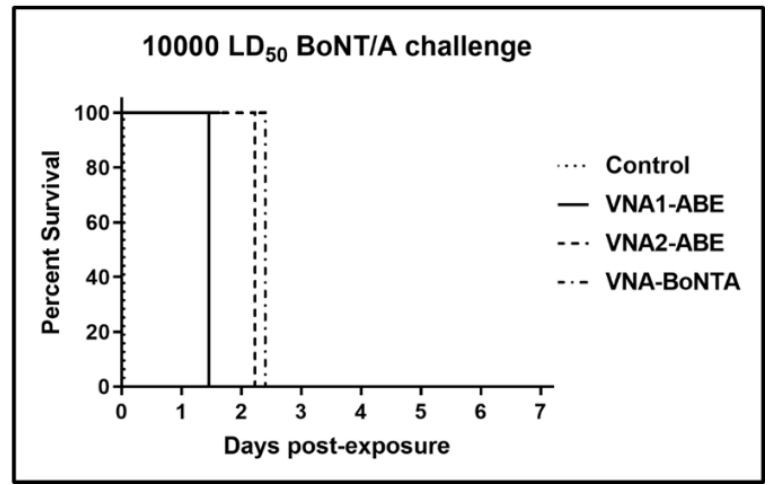

Figure 4. Heterohexamer VNAs protect mice from 500 LD 50 doses of BoNT/B and delay death from 10,000 LD 50 doses of BoNT/A. A. Groups of five mice were co-administered $2 \mu \mathrm{g}$ of VNA1-ABE, VNA2-ABE (20 pmoles), or 1 ug of VNA-BoNTB (20 pmoles) and 500 LD50 of BoNT/B. Mice were then monitored for survival and signs of botulism for a week. The survival of mice is plotted as a function of time post-exposure. All VNA-treated groups had 100\% survival, significantly different from untreated controls ( $p<0.001$ by log-rank Mantel-Cox test). B. Groups of five mice were coadministered $1 \mu \mathrm{g}$ of VNA1-ABE, VNA2-ABE (10 pmoles), or 2 ug of VNA-BoNTA (40 pmoles) and 10,000 LD50 of BoNT/A. Mice were then monitored for survival and signs of botulism. The survival of mice is plotted as a function of time post-exposure. Treated groups differed significantly from untreated controls ( $\mathrm{p}<0.01$ by log-rank Mantel-Cox test).

\section{Discussion}

VHHs have proven to be excellent components of BoNT antitoxin agents when produced as linked multimers [19, 27, 28]. The ability to express VHHs as multimers makes possible the ability to develop therapeutic antitoxins targeting multiple different toxins, such as $C$. difficile toxins TcdA and TcdB [9, 13], anthrax EF and LF [12] or Shiga toxins Stx1 and Stx2 [10], with a single protein. Here we extend these findings to show that a single heterohexameric protein containing six different linked VHH components makes possible antitoxin protection from three different BoNT serotypes. Since the heterohexamers retained the same apparent affinity and antitoxin potencies as the heterodimers for each serotype, these results also indicate that all component VHHs retain their full binding and neutralizing functions. This implies that even the VHH components located proximal to the carboxyl end of the growing chain of six linked VHHs continue to fold into independent functioning domains during translation and secretion.

Two different heterohexamer constructions were produced; one in which VHH pairs targeting the same toxin serotype were placed adjacent to one another, and the second construction in which the two VHHs targeting the same serotype were separated by two VHHs targeting a different serotype (Figure 1A). We were unable to identify any clear differences between the two different constructions in terms of their apparent affinity for their toxin targets or their ability to protect mice from intoxication by the different BoNT serotypes. A previous study has indicated that heterodimeric VNAs in which both VHH components are sterically capable of binding simultaneously to the toxin are more potent antitoxins [27]. Our results show that separating the VHH components with several bulky VHH components did not demonstrably improve the potency and thus suggest that the VHHs in this heterodimer did not permit simultaneous binding of both VHH components to the same toxin molecule despite the presence of two intervening linked VHHs. Furthermore, the fact that both VNA heterohexamers displayed the same antitoxin potencies as the individual heterodimers for each serotype strongly suggests that all six of the individual $\mathrm{VHH}$ components retained their full toxin binding functions. This is most well demonstrated for BoNT/E as both anti-BoNT/E VHH components are present at the carboxyl terminus of the heterohexamer, 
the last components synthesized during translation, and yet the heterohexamer retained the same anti-BoNT/E binding affinity by ELISA and the same antitoxin potency in vivo as the VNA/BoNTE heterodimer.

Antitoxins for botulism, or any toxin, should possess the ability to provide protection from most if not all natural variants of the toxin to which they may be exposed. Use of antitoxins that derive from polyclonal antibodies generally offer protection to natural variants because they consist of many different antibodies recognizing different toxin epitopes, thus typically having a subset of cross-specific antibodies that recognize each of the variants. But natural variants create a challenge for antitoxin products consisting of clonal antibody components. For example, monoclonal antibodies (mAbs) must be identified that broadly recognize all known natural variants or, alternatively, pools of mAbs must be produced that, in combination, provide broad variant protection. The ability to produce long chains of $\mathrm{VHH}$ components, up to at least six different VHHs as shown here, offers an alternative solution as it permits the incorporation of numerous VHHs into one or a few proteins designed to neutralize virtually all of the natural diversity of the toxin target. While such an idealized goal is particularly challenging for a botulism antitoxin, we suggest that it should be possible to produce a broad BoNT antitoxin consisting of perhaps only three or four heteromultimeric proteins. This would be accomplished by selecting the appropriate BoNT-neutralizing VHH components such that all naturally occurring BoNT serotypes and subtypes are targeted for neutralization by at least two of the VHH components.

One attractive option for a next-generation BoNT antitoxin might be nanoparticle-encapsulated RNA encoding three or four heteromultimer antitoxin proteins that together possess good potency to all known BoNT serotypes and subtypes in nature. We have reported the successful use of RNA nanoparticles to elicit high levels of expression of antitoxin VNAs in mice [15] as a novel form of passive immunization. Mice were shown to express VNA serum levels that exceeded $100 \mathrm{ug} / \mathrm{ml}$ at day 1 post treatment and fully protected mice from lethal challenges of either BoNT/A or BoNT/B in mice. Prior studies have shown that only about $10 \mathrm{ng}$ of VNA is sufficient to protect mice from 10 $\mathrm{LD}_{50}$ of BoNT/A [7] which is 10,000-fold below the levels produced in the RNA treated mice. More importantly, the serum VNAs were easily detectable in mice within 2 hours post-treatment and the post-intoxication treatment window was indistinguishable between mice treated with RNA nanoparticles and those given intravenous protein VNA [15].

\section{Conclusions}

We have demonstrated that VHH heteromultimers of six VHHs can be efficiently expressed and secreted in mammalian cells with the retention of the full function of each VHH component. We also demonstrate that a single $\mathrm{VHH}$ heterohexamer can be produced that effectively protects animals from intoxication by three different toxins, in this case BoNT/A, BoNT/B and BoNT/E. These findings suggest the feasibility of developing a broad specificity BoNT antitoxin capable of treating recent exposures from virtually any natural BoNT serotype or subtype with a small pool of VHH multimers. Furthermore, this work highlights the great potential of VHH antibodies to retain high their individual functionality even when expressed as chains of linked VHHs containing at least six VHH components.

\section{Materials and Methods}

\subsection{Ethics statement}

All in vivo studies were performed within the guidelines established by the Guide for the Care and Use of Laboratory Animals of the National Institutes of Health and were approved by the Tufts 
BoNT/A, BoNT/B and BoNT/E complex preparations (subtype 1 for each toxin, all from Metabiologics Inc.) were used for in vivo assays. Prior to use, BoNT/E was subjected to trypsin activation. Briefly, $25 \mathrm{ul} 1.2 \mathrm{mg} / \mathrm{ml}$ trypsin (EMD \#650211) in HEPES (Sigma \#H0887), $50 \mathrm{ul} 1 \mathrm{mg} / \mathrm{ml}$ Complexed Botulinum Toxin E and 100 ul sterile PBS were incubated $30 \mathrm{~min}$. at $37^{\circ} \mathrm{C}$. Following incubation, $25 \mathrm{ul} 2.5 \mathrm{mg} / \mathrm{ml}$ trypsin inhibitor (Sigma \#T6522) was added and the mixture further incubated $15 \mathrm{~min}$. at room temperature. The resulting solution containing $0.25 \mathrm{ug} / \mathrm{ul}$ activated BoNT/E was stored at $-80^{\circ} \mathrm{C}$ until use. BoNT/A, BoNT/B and the activated BoNT/E were carefully titrated in vivo to determine the LD50 prior to use in assays performed for VHH evaluation. All procedures utilizing BoNT were performed within a CDC-registered Select Agent laboratory.

\subsection{VHH and VNA expression}

VHHs and some VHH heterodimeric VNAs were expressed in E. coli as previously reported. For expression of most VNAs, a CHO cell host was employed. Coding DNA encoding the VNAs was synthesized by Genscript with restriction sites compatible with insertion in frame into a mammalian expression vector based on pSecTag2. The sequences of encoded heterohexamer proteins are shown in Supplemental Figure S1. Similar expression vectors were engineered for expression of heterodimeric VNAs encoding VHHs A1/A2 (VNA-BoNTA) or B1/B2 (VNA-BoNTB). Expression of the heterodimeric VNA-BoNTE containing E1/E2 is described as Trx/E/JLE-G6/JLEE9/E in Tremblay et al [28] and was produced and purified from E. coli. VNAs contained E-tags tags for detection. The mammalian expression plasmids were transfected into CHO-S cells in suspension cultures of serum-free FreeStyle ${ }^{\mathrm{TM}} \mathrm{CHO}$ cell medium. Cells were transfected using FreeStyle ${ }^{\mathrm{TM}}$ MAX reagent (Invitrogen) according to the manufacturer's recommendations and incubated under orbital shaking for three days. Conditioned medium was harvested under sterile conditions and stored at $4^{\circ} \mathrm{C}$. VHH and VNA expression levels were estimated by comparison to protein standards using BioRad Image Lab software.

\subsection{ELISAs}

ELISAs were performed using Costar tissue culture 96 well plates to reduce the conformational deformation that occurs with conventional ELISA plastic [28]. Catalytically inactive BoNT proteins representing BoNT/A, BoNT/B and BoNT/E [29-31] (kindly provided by Dr. Robert Webb, USAMRICD) were coated at $4^{\circ} \mathrm{C}$ overnight at $1 \mu \mathrm{g} / \mathrm{ml}$ in PBS, then blocked for at least an hour at $37^{\circ} \mathrm{C}$ with $4 \%$ milk in PBS, $0.1 \%$ Tween. After washing, dilution ELISAs were initiated by diluting the VNAs to $25 \mathrm{nM}$ and performing serial dilutions of $1: 5$. After incubation for one hour at $37{ }^{\circ} \mathrm{C}$, plates were washed and then incubated with 1:10,000 rabbit HRP/anti-E-tag (Bethyl) for one hour, washed, developed with TMB (Sigma) as recommended by manufacturer and read at A450.

\subsection{Standard mouse toxin lethality assay}

VNA1-ABE and VNA2-ABE were evaluated in vivo using the BoNT murine lethality assay. The lethality assays utilized adult, $\sim 20 \mathrm{~g}$ female CD-1 mice (Charles River Labs) housed in standard shoebox cages at 5 mice/cage. Following arrival, mice were observed at least twice daily and cages containing corncob bedding and nestlets along with standard rodent chow within the cage rack and water ad libitum were changed twice weekly. Throughout each study, mice were also offered food on the cage bottom along with both nutritional (Napa Nectar; Lenderking \#88-0001) and hydration (Boost; Clear H2O \#72-04-5022) supplement gels. One day prior to initiation of each study, mice were weighed and sorted to reduce intergroup $(n=5)$ weight variation. VNA-BoNT mixtures were co-administered IV via the lateral tail vein within a 200-250 $\mu$ l volume. Each mouse within the respective group received $1 \mu \mathrm{g}$ of VNA1-ABE (10 pmoles) or VNA2-ABE (10 pmoles) and $100 \mathrm{LD}_{50}$ of BoNT/A1, BoNT/B1 or BoNT/E or 100 LD50 BoNT/A1, BoNT/B1 and BoNT/E. Following 
administration of BoNT, mice were observed a minimum of four times each day between 9 a.m. and midnight with additional checks throughout peak periods of mortality to ensure accurate documentation of time to death data. Time to death was defined as the time at which a mouse was found dead or was euthanized due to severe morbidity. At each check, a standard score sheet was utilized to objectively document the overall disposition and clinical symptoms exhibited by each mouse. Mice which were observed to be open-mouth breathing, lethargic, unresponsive or moribund were euthanized via carbon dioxide asphyxiation followed by cervical dislocation.

Supplementary Materials: The following are available online at www.mdpi.com/xxx/s1, Figure S1: Sequences of heterohexamer VNAs and their component VHHs.

Author Contributions: Conceptualization, C.B.S., methodology, J.M., J.M.T, M.D., A.F., J.A, formal analysis J.M., C.B.S., investigation, J.M., J.M.T, M.D., A.F., J.A, data curation, J.M., J.M.T, M.D., A.F, supervision, J.M., C.B.S., writing, J.M., C.B.S., funding acquisition, C.B.S. All authors have read and agreed to the published version of the manuscript.

Funding: This research was funded in part by NIH grant R01AI125704 and R01AI093467 (CBS) from NIH NIAID and DARPA HROO11-14-2-0005 subaward 10-1798-9902. The content is solely the responsibility of the authors and does not necessarily represent the official views of the National Institute of Allergy and Infectious Diseases or the National Institutes of Health.

Acknowledgments: We gratefully acknowledge Karen Baldwin, Kwasi Ofori and Zachary Forbes for expert technical assistance with handling of alpacas, isolation of PBLs and performance of mouse LD50 assays which made these subsequent studies possible. We are also grateful to Dr. Patrick M. McNutt for help with some of the statistical analysis.

Conflicts of Interest: The authors declare no conflict of interest. The funders had no role in the design of the study; in the collection, analyses, or interpretation of data; in the writing of the manuscript, or in the decision to publish the results.

\section{References}

1. Muyldermans S. Nanobodies: natural single-domain antibodies. Annu Rev Biochem. 2013;82:775-97. doi: 10.1146/annurev-biochem-063011-092449. PubMed PMID: 23495938.

2. Konning D, Zielonka S, Grzeschik J, Empting M, Valldorf B, Krah S, et al. Camelid and shark single domain antibodies: structural features and therapeutic potential. Curr Opin Struct Biol. 2017;45:10-6. Epub 2016/11/20. doi: 10.1016/j.sbi.2016.10.019. PubMed PMID: 27865111.

3. Arbabi Ghahroudi M, Desmyter A, Wyns L, Hamers R, Muyldermans S. Selection and identification of single domain antibody fragments from camel heavy-chain antibodies. FEBS Lett. 1997;414(3):521-6. PubMed PMID: 9323027.

4. Dumoulin M, Conrath K, Van Meirhaeghe A, Meersman F, Heremans K, Frenken LG, et al. Single-domain antibody fragments with high conformational stability. Protein Sci. 2002;11(3):50015. PubMed PMID: 11847273.

5. van der Linden RH, Frenken LG, de Geus B, Harmsen MM, Ruuls RC, Stok W, et al. Comparison of physical chemical properties of llama VHH antibody fragments and mouse monoclonal antibodies. Biochim Biophys Acta. 1999;1431(1):37-46. PubMed PMID: 10209277. 6. Moayeri M, Leysath CE, Tremblay JM, Vrentas C, Crown D, Leppla SH, et al. A Heterodimer of a VHH Antibody that Inhibits Anthrax Toxin Cell Binding Linked to a VHH that Blocks Oligomer Formation is Highly Protective in an Anthrax Spore Challenge Model. The Journal of biological chemistry. 2015. doi: 10.1074/jbc.M114.627943. PubMed PMID: 25564615. 7. Mukherjee J, Dmitriev I, Debatis M, Tremblay JM, Beamer G, Kashentseva EA, et al. Prolonged prophylactic protection from botulism with a single adenovirus treatment promoting serum 
expression of a VHH-based antitoxin protein. PloS one. 2014;9(8):e106422. doi: 10.1371/journal.pone.0106422. PubMed PMID: 25170904; PubMed Central PMCID: PMC4149568. 8. Vance DJ, Tremblay JM, Mantis NJ, Shoemaker CB. Stepwise engineering of heterodimeric single domain camelid $\mathrm{VHH}$ antibodies that passively protect mice from ricin toxin. The Journal of biological chemistry. 2013;288(51):36538-47. doi: 10.1074/jbc.M113.519207. PubMed PMID: 24202178; PubMed Central PMCID: PMC3868766.

379 9. Schmidt DJ, Beamer G, Tremblay JM, Steele JA, Kim HB, Wang Y, et al. A Tetraspecific VHH-

380 based Neutralizing Antibody Modifies Disease Outcome in Three Animal Models of Clostridium

381 difficile Infection. Clin Vaccine Immunol. 2016. doi: 10.1128/CVI.00730-15. PubMed PMID:

$382 \quad 27413067$.

383 10. Sheoran AS, Dmitriev IP, Kashentseva EA, Cohen O, Mukherjee J, Debatis M, et al. Adenovirus

384 vector expressing Stx1/Stx2-neutralizing agent protects piglets infected with Escherichia coli O157:H7 against fatal systemic intoxication. Infection and immunity. 2015;83(1):286-91. doi:

386 10.1128/IAI.02360-14. PubMed PMID: 25368111; PubMed Central PMCID: PMC4288880. 11. Tremblay JM, Mukherjee J, Leysath CE, Debatis M, Ofori K, Baldwin K, et al. A single VHHbased toxin-neutralizing agent and an effector antibody protect mice against challenge with Shiga toxins 1 and 2. Infection and immunity. 2013;81(12):4592-603. doi: 10.1128/IAI.01033-13. PubMed PMID: 24082082; PubMed Central PMCID: PMC3837998.

391 12. Vrentas CE, Moayeri M, Keefer AB, Greaney AJ, Tremblay J, O'Mard D, et al. A diverse set of

392 single-domain antibodies (VHHs) against the anthrax toxin lethal and edema factors provides a 393 basis for construction of a bispecific agent that protects against anthrax infection. The Journal of 394 biological chemistry. 2016. doi: 10.1074/jbc.M116.749184. PubMed PMID: 27539858.

395 13. Yang Z, Schmidt D, Liu W, Li S, Shi L, Sheng J, et al. A novel multivalent, single-domain 396 antibody targeting TcdA and TcdB prevents fulminant Clostridium difficile infection in mice. J 397 Infect Dis. 2014. doi: 10.1093/infdis/jiu196. PubMed PMID: 24683195.

398 14. Terryn S, Francart A, Lamoral S, Hultberg A, Rommelaere H, Wittelsberger A, et al. Protective 399 effect of different anti-rabies virus VHH constructs against rabies disease in mice. PloS one.

400 2014;9(10):e109367. Epub 2014/10/28. doi: 10.1371/journal.pone.0109367. PubMed PMID: 25347556;

401 PubMed Central PMCID: PMCPMC4210127.

402 15. Thran M, Mukherjee J, Ponisch M, Fiedler K, Thess A, Mui BL, et al. mRNA mediates passive 403 vaccination against infectious agents, toxins, and tumors. EMBO Mol Med. 2017. doi:

404 10.15252/emmm.201707678. PubMed PMID: 28794134; PubMed Central PMCID: PMCPMC5623855.

405 16. Sepulveda J, Mukherjee J, Tzipori S, Simpson LL, Shoemaker CB. Efficient serum clearance of 406 botulinum neurotoxin achieved using a pool of small antitoxin binding agents. Infection and 407 immunity. 2010;78(2):756-63. Epub 2009/11/18. doi: 10.1128/IAI.01084-09. PubMed PMID: 19917718; 408 PubMed Central PMCID: PMC2812214.

409 17. Liu L. Pharmacokinetics of monoclonal antibodies and Fc-fusion proteins. Protein Cell. 410 2018;9(1):15-32. Epub 2017/04/20. doi: 10.1007/s13238-017-0408-4. PubMed PMID: 28421387; PubMed 411 Central PMCID: PMCPMC5777971.

412 18. Richard G, Meyers AJ, McLean MD, Arbabi-Ghahroudi M, MacKenzie R, Hall JC. In vivo 413 neutralization of alpha-cobratoxin with high-affinity llama single-domain antibodies (VHHs) and a 414 VHH-Fc antibody. PloS one. 2013;8(7):e69495. Epub 2013/07/31. doi: 10.1371/journal.pone.0069495. 415 PubMed PMID: 23894495; PubMed Central PMCID: PMCPMC3718736. 
19. Mukherjee J, Tremblay JM, Leysath CE, Ofori K, Baldwin K, Feng X, et al. A novel strategy for development of recombinant antitoxin therapeutics tested in a mouse botulism model. PloS one. 2012;7(1):e29941. Epub 2012/01/13. doi: 10.1371/journal.pone.0029941. PubMed PMID: 22238680. 20. Maslanka SE, Luquez C, Dykes JK, Tepp WH, Pier CL, Pellett S, et al. A Novel Botulinum Neurotoxin, Previously Reported as Serotype H, Has a Hybrid-Like Structure With Regions of Similarity to the Structures of Serotypes A and F and Is Neutralized With Serotype A Antitoxin. J Infect Dis. 2016;213(3):379-85. Epub 2015/06/13. doi: 10.1093/infdis/jiv327. PubMed PMID: 26068781; PubMed Central PMCID: PMCPMC4704661.

21. Nayak SU, Griffiss JM, McKenzie R, Fuchs EJ, Jurao RA, An AT, et al. Safety and pharmacokinetics of $\mathrm{XOMA} 3 \mathrm{AB}$, a novel mixture of three monoclonal antibodies against botulinum toxin A. Antimicrob Agents Chemother. 2014;58(9):5047-53. Epub 2014/06/11. doi: 10.1128/AAC.02830-14. PubMed PMID: 24913160; PubMed Central PMCID: PMCPMC4135817. 22. Meng Q, Garcia-Rodriguez C, Manzanarez G, Silberg MA, Conrad F, Bettencourt J, et al. Engineered domain-based assays to identify individual antibodies in oligoclonal combinations targeting the same protein. Anal Biochem. 2012;430(2):141-50. Epub 2012/08/28. doi: 10.1016/j.ab.2012.08.005. PubMed PMID: 22922799; PubMed Central PMCID: PMCPMC4209713. 23. Garcia-Rodriguez C, Razai A, Geren IN, Lou J, Conrad F, Wen WH, et al. A Three Monoclonal Antibody Combination Potently Neutralizes Multiple Botulinum Neurotoxin Serotype E Subtypes. Toxins. 2018;10(3). Epub 2018/03/02. doi: 10.3390/toxins10030105. PubMed PMID: 29494481; PubMed Central PMCID: PMCPMC5869393. 24. Fan Y, Garcia-Rodriguez C, Lou J, Wen W, Conrad F, Zhai W, et al. A three monoclonal antibody combination potently neutralizes multiple botulinum neurotoxin serotype $\mathrm{F}$ subtypes. PloS one. 2017;12(3):e0174187. Epub 2017/03/23. doi: 10.1371/journal.pone.0174187. PubMed PMID: 28323873; PubMed Central PMCID: PMCPMC5360321. 25. Fan Y, Barash JR, Lou J, Conrad F, Marks JD, Arnon SS. Immunological Characterization and Neutralizing Ability of Monoclonal Antibodies Directed Against Botulinum Neurotoxin Type H. J Infect Dis. 2016;213(10):1606-14. Epub 2016/03/05. doi: 10.1093/infdis/jiv770. PubMed PMID: 26936913; PubMed Central PMCID: PMCPMC4837907. Combinations Prevent Serotype A and Serotype B Inhalational Botulism in a Guinea Pig Model. Toxins. 2019;11(4). Epub 2019/04/10. doi: 10.3390/toxins11040208. PubMed PMID: 30959899. 27. Lam KH, Tremblay JM, Vazquez-Cintron E, Perry K, Ondeck C, Webb RP, et al. Structural Insights into Rational Design of Single-Domain Antibody-Based Antitoxins against Botulinum Neurotoxins. Cell Rep. 2020;30(8):2526-39 e6. Epub 2020/02/27. doi: 10.1016/j.celrep.2020.01.107. 450 PubMed PMID: 32101733.

451 28. Tremblay JM, Vazquez-Cintron E, Lam KH, Mukherjee J, Bedenice D, Ondeck CA, et al. 452 Camelid VHH Antibodies that Neutralize Botulinum Neurotoxin Serotype E Intoxication or 453 Protease Function. Toxins. 2020;12(10). Epub 2020/09/30. doi: 10.3390/toxins12100611. PubMed 454 PMID: 32987745; PubMed Central PMCID: PMCPMC7598594.

455 29. Webb RP, Smith TJ, Smith LA, Wright PM, Guernieri RL, Brown JL, et al. Recombinant 456 Botulinum Neurotoxin Hc Subunit (BoNT Hc) and Catalytically Inactive Clostridium botulinum 457 Holoproteins (ciBoNT HPs) as Vaccine Candidates for the Prevention of Botulism. Toxins. 2017;9(9). 
458 Epub 2017/09/05. doi: 10.3390/toxins9090269. PubMed PMID: 28869522; PubMed Central PMCID: 459 PMCPMC5618202.

460 30. Webb RP, Smith TJ, Wright P, Brown J, Smith LA. Production of catalytically inactive BoNT/A1 461 holoprotein and comparison with BoNT/A1 subunit vaccines against toxin subtypes A1, A2, and 462 A3. Vaccine. 2009;27(33):4490-7. Epub 2009/05/20. doi: S0264-410X(09)00705-1 [pii]

463 10.1016/j.vaccine.2009.05.030. PubMed PMID: 19450643.

464 31. Webb R, Wright PM, Brown JL, Skerry JC, Guernieri RL, Smith TJ, et al. Potency and stability 465 of a trivalent, catalytically inactive vaccine against botulinum neurotoxin serotypes $\mathrm{C}, \mathrm{E}$ and $\mathrm{F}$ 466 (triCEF). Toxicon. 2020;176:67-76. Epub 2020/02/08. doi: 10.1016/j.toxicon.2020.02.001. PubMed 467 PMID: 32032587. 